\title{
一种新的可控/活性自由基聚合方法一DPETTX调 节苯乙烯自由基聚合研究
}

\author{
王世超 ${ }^{1,2}$, 王力 ${ }^{1,2}$, 于昊宇 ${ }^{1,2}$, 邵健为 ${ }^{1,2}$, 杨万泰 ${ }^{1,2^{*}}$ \\ 1. 北京化工大学, 化工资源有效利用国家重点实验室, 北京 100029 \\ 2. 北京化工大学材料科学与工程学院, 北京 100029 \\ *通讯作者, E-mail: yangwt@mail.buct.edu.cn \\ 收稿日期：2017-12-05; 接受日期：2018-03-06; 网络版发表日期：2018-05-23 \\ 国家自然科学基金(编号: 21404004)资助项目
}

摘要可控/活性自由基聚合(CLRP)自20世纪末以来获得了非常迅猛的发展，但由于已有方法的缺陷和局限， 限制了其工业化规模应用。因此，开发可控、活性程度更高、更加简单实用且环境友好的CLRP新方法具有重要 意义。本文制备了一种芳香环状硫醚化合物9-(1-苯乙基)-9-((1-苯乙基)硫基)-9H-硫杂葱DPETTX，对其单独引发 和与少量偶氮二异庚腈 $(\mathrm{ABVN})$ 共同引发苯乙烯 $(\mathrm{St})$ 聚合的行为进行了系统评价. 结果表明, DPETTX可在高温 $\left(100^{\circ} \mathrm{C}\right)$ 下单独引发 $\mathrm{St}$ 聚合, 但引发效率较低; 当其与少量 $\mathrm{ABVN}$ 共用时, 可使 $\mathrm{St}$ 在低温下 $\left(55^{\circ} \mathrm{C}\right)$ 聚合, 聚苯乙烯 $(\mathrm{PS})$ 的数均分子量 $\left(M_{\mathrm{n}}\right)$ 随转化率升高而不断增大, 如摩尔比ABVN/DPETTX $=0.05 / 1$ 时, $M_{\mathrm{n}}$ 从 2.7 万增长到 4.6 万. 该 基于DPETTX和少量ABVN的共引发体系, 打开了一条开发新可控/活性自由基聚合体系的路径.

关键词硫醚, 自由基, 聚合, 分子量

\section{1 引言}

可控/活性自由基聚合(CLRP)是大分子合成的强 大工具，其不仅可以合成分子量可控且具有窄分布的 大分子链，而且可以实现具有不同组成、拓扑结构和 官能团的大分子链的精确高效制备. 因其具有诸多优 势, CLRP在生物医学、分析检测、光电和纳米复合 材料等领域的研究中获得了十分广泛的应用 ${ }^{[1]}$. 氮氧

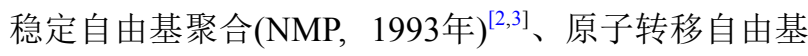
聚合(ATRP，1995年) ${ }^{[4,5]}$ 和可逆加成-断裂链转移自由 基聚合(RAFT, 1998年) ${ }^{[6]}$ 是 3 种代表性的CLRP方法. 经
过数十年的发展, 这些方法不断得到改进且应用领域 日益拓宽. 在其之后，还涌现出了单电子转移自由基 聚合(SET-LRP) ${ }^{[7]}$ 和碲、锑或铋调节的自由基聚合 ${ }^{[8 \sim 10]}$ 等形形色色的CLRP新方法，但这些方法存在条件苛 刻、适用范围小和含有毒过渡金属等问题，导致至今 未能进入规模工业应用. 因此, 开发适用性强、体系 简单且环境友好的CLRP新方法, 仍然是该领域的一 大研究热点.

本实验室长期致力于CLRP新方法的开发, 已经开 发出“环状频哪醇自由基调节的CLRP”方法 ${ }^{[1113]}$, 该方 法的核心是芳香频哪醇自由基对聚合物链自由基的可

引用格式: Wang S, Wang L, Yu H, Shao J, Yang W. Radical polymerization of styrene mediated by 9-(1-phenylethyl)-9-((1-phenylethyl)thio)-9H-thioxanthene. Sci Sin Chim, 2018, 48: 601-608, doi: 10.1360/N032017-00204 
逆活化/针化使聚合物分子链持续增长. 近年来，我们 结合澳洲Rizzardo 等 ${ }^{[14]}$ 提出的硫酮加成链转移控制聚 合物分子量的方法，以及Barner-Kowollik等 ${ }^{[15 \sim 18]}$ 提出 的“硫酮调节的自由基聚合”方法，进一步开发了氧 (硫)杂葱-9-硫酮调节的CLRP聚合新体系。从中发现, 链自由基向氧(硫)杂葱-9-硫酮加成形成的稳定自由基 可与另一链自由基发生交叉终止，该交叉终止产物在 聚合过程中通过可逆断裂/偶合控制聚合物链的增长. 为此，本文设计合成了与该交叉终止产物具有类似结 构的硫醚分子9-(1-苯乙基)-9-((1-苯乙基)硫基)-9H-硫 杂葱DPETTX, 并对其调节苯乙烯(St)自由基聚合的行 为进行了系统研究. 发现将DPETTX与少量普通自由 基引发剂并用, St聚合体系表现出一定的可控性, 聚合 产物的数均分子量 $\left(M_{\mathrm{n}}\right)$ 随转化率呈现明显的增长. 这 些结果为认识该新体系的反应机理以及对其进行进一 步改进和完善奠定了基础.

\section{2 实验部分}

\section{1 试剂}

硫杂葱酮: Alfa Aesar (中国)化学有限公司, 分析 纯; 劳森试剂: Alfa Aesar (中国)化学有限公司, 97\%; 甲苯: 北京化工厂 (中国), 蒸馏处理; 碳酸氢钠: 天津市 光复科技发展有限公司(中国)，分析纯；乙酸乙酯：天 津市光复科技发展有限公司(中国), 分析纯; 1-澳苯乙 烷: Alfa Aesar (中国)化学有限公司, 97\%; 纳米铜粉： 北京伊诺凯科技有限公司(中国), $98 \%$; 五甲基二乙烯 三胺: PMDETA, 北京伊诺凯科技有限公司, $98 \%$; 二氯 甲烷: 北京化工厂, 分析纯; 石油醚: 北京化工厂, 分析 纯; 偶氮二异庚腈: ABVN, 上海麦克林生化科技有限 公司(中国), 98\%; 苯乙烯: 国药集团(中国), 减压蒸馏 后使用.

\section{2 测试与表征}

采用核磁共振谱仪(Bruker AV600, 德国)进行核磁 共振氢谱 $\left({ }^{1} \mathrm{H}\right.$ NMR) 分析，表征所合成小分子的结构. 采用 $\mathrm{CDCl}_{3}$ 或 $\mathrm{CD}_{3} \mathrm{COCD}_{3}$ 作为溶剂，四甲基硅烷(TMS) 为内标.

采用紫外-可见分光光度计 (Hitachi U3900H，日 本)对所合成小分子进行紫外-可见光谱(UV-Vis)分析,
以二氯甲烷作为溶剂.

采用称重法计算聚合反应中单体的转化率，计算 公式如下:

$$
C=\frac{m_{3}-m_{1}}{\left(m_{2}-m_{1}\right) \times C_{0}},
$$

其中, $m_{1} 、 m_{2}$ 和 $m_{3}$ 分别为称量瓶、称量瓶 + 取出样品 和称量瓶+干燥后样品的质量, $C_{0}$ 为初始状态体系中 单体的质量分数.

采用凝胶渗透色谱仪(GPC, HPLC/GPC515)测定 所得聚苯乙烯 $(\mathrm{PS})$ 的数均分子量 $\left(M_{\mathrm{n}}\right)$ 和多分散性指数 (PDI). 以单分散聚苯乙烯作为标样校正，采用交联聚 苯乙烯(styragelHT6E+HT5+HT3)色谱柱，淋洗液为四 氢呋喃.

\subsection{DPETTX的合成}

将 $2.12 \mathrm{~g}$ 硫杂葱酮 $(10 \mathrm{mmol}) 、 2.42 \mathrm{~g}$ 劳森试剂 (12 mmol)和 $30 \mathrm{~mL}$ 甲苯加入到 $50 \mathrm{~mL}$ 圆底烧瓶中, 在温 度为 $75^{\circ} \mathrm{C}$ 的恒温摚拌浴中反应过夜. 旋转蒸发除去溶 剂，用 $300 \mathrm{~mL}$ 饱和 $\mathrm{NaHCO}_{3}$ 水溶液分 3 次洗涤剩余固 体，除去残余的劳森试剂. 然后用乙酸乙酯溶解洗涤 后的固体, 过滤除去不溶性杂质, 再次旋干溶剂, 并用 甲苯重结晶, 得到 $1.6 \mathrm{~g}$ 褐色针状的硫杂葱-9-硫酮晶体, 收率约为70\%. ${ }^{1} \mathrm{H} \mathrm{NMR}\left(\mathrm{CDCl}_{3}, 600 \mathrm{MHz}, \delta, \mathrm{ppm}\right)$ : $8.78(\mathrm{~d}, 2 \mathrm{H}), 7.76(\mathrm{t}, 3 \mathrm{H}), 7.53(\mathrm{~d}, 2 \mathrm{H}), 7.40(\mathrm{t}, 3 \mathrm{H})$. UVVis吸收峰(nm): 312, 406.

将 $1.5 \mathrm{~g}$ (6.6 mmol)的硫杂葱-9-硫酮、 $2.44 \mathrm{~g}$ (13.2 mmol) 1 -溴苯乙烷、 $0.84 \mathrm{~g}(13.2 \mathrm{mmol})$ 纳米铜 粉、 $2.28 \mathrm{~g}$ (13.2 mmol) PMDETA和 $8 \mathrm{~mL}$ 甲苯加入到 $25 \mathrm{~mL}$ 带支管的圆底烧瓶中, 通过3次冷冻抽排排除体 系中的氧气并充入氮气. 将反应瓶置于温度为 $60^{\circ} \mathrm{C}$ 的 恒温搅拌浴中，反应过夜后旋干体系，加入二氯甲烷 溶解并用去离子水多次洗涤以除去大部分的铜络合 物. 然后, 经柱层析分离(展开剂: 二氯甲烷/石油醚= $1 / 9)$, 得到 $1.0 \mathrm{~g}$ 淡青色的DPETTX固体, 收率约为 $23 \%$.

${ }^{1} \mathrm{H}$ NMR $\left(\mathrm{CD}_{3} \mathrm{COCD}_{3}, 600 \mathrm{MHz}, \delta, \mathrm{ppm}\right): 8.18(\mathrm{~d}, 1 \mathrm{H})$, $7.82(\mathrm{~d}, 1 \mathrm{H}), 7.71(\mathrm{~d}, 1 \mathrm{H}), 7.41 \sim 7.35(\mathrm{~m}, 2 \mathrm{H}), 7.29$ (t, 1H), $7.25(\mathrm{t}, 1 \mathrm{H}), 7.17 \sim 7.13(\mathrm{~m}, 2 \mathrm{H}), 7.08 \sim 6.98(\mathrm{~m}, 9 \mathrm{H})$, 6.97 6.87 (m, 6H), 6.83 6.73 (m, 7H), $6.66(\mathrm{~d}, 1 \mathrm{H}), 3.50$ (quint, 2H), 3.38 (quint, 2H), 1.26 (q, 6H), 1.19 (q, 6H). UV-Vis吸收峰(nm): 280, 301. 


\section{4 聚合反应}

精确称取需要量的ABVN、DPETTX、St和溶剂 加入 $25 \mathrm{~mL}$ 带支管的圆底烧瓶，通过3次冷冻抽排排除 体系中的氧气并充入氮气. 将反应瓶置于设置好温度 的恒温搅拌浴中进行反应，每隔一定时间从中取出少 量样品加入称量瓶, 用于转化率、 $M_{\mathrm{n}}$ 及PDI的测定. 聚 合反应配方及结果见表 $\mathrm{S} 1$ (网络版补充材料).

\section{3 结果与讨论}

\subsection{DPETTX的合成及表征}

我们设计的DPETTX的结构式及合成反应式如图 1所示. 为合成该未见报道的DPETTX分子，我们尝试 了各种方法，最后确定了如图1所示的反应路线，其优 化的反应配方和反应-分离方法见实验部分. 该合成路 线的主要反应机理为 1 -溴苯乙烷在 $\mathrm{Cu}$ 和PMDETA形成 的络合物的催化下脱除卤原子，形成1-苯乙基自由基; 因为硫酮具有很强的自旋捕捉能力，1-苯乙基自由基 快速向硫杂葱-9-硫酮碳硫双键的硫原子加成，形成稳 定的叔碳自由基; 该叔碳自由基与另一个 1 -苯乙基自 由基发生偶合, 得到目标产物DPETTX.

图2给出了DPETTX的 ${ }^{1} \mathrm{H}$ NMR图, 其中 $8.18 、 7.82$ 和7.71 ppm处三组两重峰对应硫杂葸环上的质子，其 峰面积比为 $1: 1: 1$, 进一步积分得到芳环上质子总数为 36. 3.50 和 $3.38 \mathrm{ppm}$ 处出现两组五重峰, 对应质子数均 为 2 个, 而 1.26 和 $1.19 \mathrm{ppm}$ 处出现两组四重峰, 对应质子 数均为 6 个. 根据质子数和各峰裂分情况推断, 产物中 存在物质的量之比为 $1: 1$ 的两组立体异构体，这源于图 1中所示的两个手性碳. 不同的立体异构体中硫杂葱环 上同一位置质子所处化学环境不同，因而其化学位移 有明显差异. 另外, 3.50 和 $3.38 \mathrm{ppm}$ 处的两组五重峰分 别由不同立体异构体中 $\mathrm{o}$ 和 $\mathrm{q}$ 的两组四重峰重叠而成, 而1.26和1.19 ppm处的两组四重峰则对应不同立体异 构体中 $\mathrm{p}$ 和 $\mathrm{r}$ 的两组两重峰. 通过二维核磁共振谱如 (COSY等, 结果未列出), 我们确定了这一推断.

\subsection{DPETTX引发St自由基聚合}

我们首先研究了DPETTX单独引发St自由基聚 合的行为. 以甲苯作为溶剂, 固定单体浓度为 $30 \mathrm{wt} \%$, DPETTX的浓度分别为 $0.43 \mathrm{wt} \%$ 和 $1.29 \mathrm{wt} \%$ 时, 在 $80^{\circ} \mathrm{C}$
和 $90^{\circ} \mathrm{C}$ 下聚合 $60 \mathrm{~h}$, 发现St转化率均低于 $20 \%$. 随后, 升 高温度至 $100^{\circ} \mathrm{C}$, 聚合速度明显提高, St转化率、 $\mathrm{PS}$ 的 $M_{\mathrm{n}}$ 和PDI的变化情况如图3所示. 由图3(a)可以看出, 随 聚合反应时间延长, St转化率逐渐升高. 当DPETTX浓 度为 $0.43 \mathrm{wt} \%$ 时, $6 \mathrm{~h}$ 的 $\mathrm{St}$ 转化率约为 $7 \%$, 而 $48 \mathrm{~h}$ 时 $\mathrm{St}$ 转 化率约为 $19 \%$; 当DPETTX浓度升高至 $1.29 \mathrm{wt} \%$ 时, $9 \mathrm{~h}$ 的 $\mathrm{St}$ 转化率约为 $15 \%$, 而 $48 \mathrm{~h}$ 的 $\mathrm{St}$ 转化率达到了 $40 \%$. 就 $M_{\mathrm{n}}$ 和PDI而言(图3(b)), 可以看出, 当DPETTX浓度 为 $0.43 \mathrm{wt} \%$ 时, 聚合过程中PS的 $M_{\mathrm{n}}$ 随转化率的升高从 14.4 万降低到 8.3 万，PDI从 1.73 升高到 2.43 ; 而当 DPETTX浓度升高到 $1.29 \mathrm{wt} \%$ 时, PS的 $M_{\mathrm{n}}$ 从 5.4 万降低 到 3.3 万, 其PDI也较宽, 从 2.37 升高至 2.73 . 实际上, 以 引发剂效率 $100 \%$ 计算时, DPETTX的浓度为 $0.43 \mathrm{wt} \%$ 和 $1.29 \mathrm{wt} \%$ 时的设计分子量分别为 3 万和 1 万，很显然 大部分的DPETTX在此聚合温度下并未分解. 综合来 看, DPETTX在高温下可单独作为引发剂引发 $\mathrm{St}$ 的自

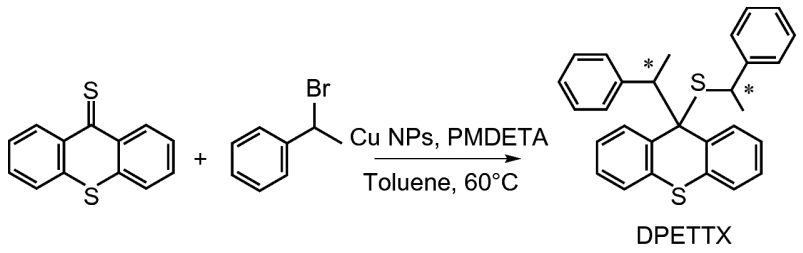

图 1 DPETTX的合成反应式

Figure 1 Synthetic route to DPETTX.

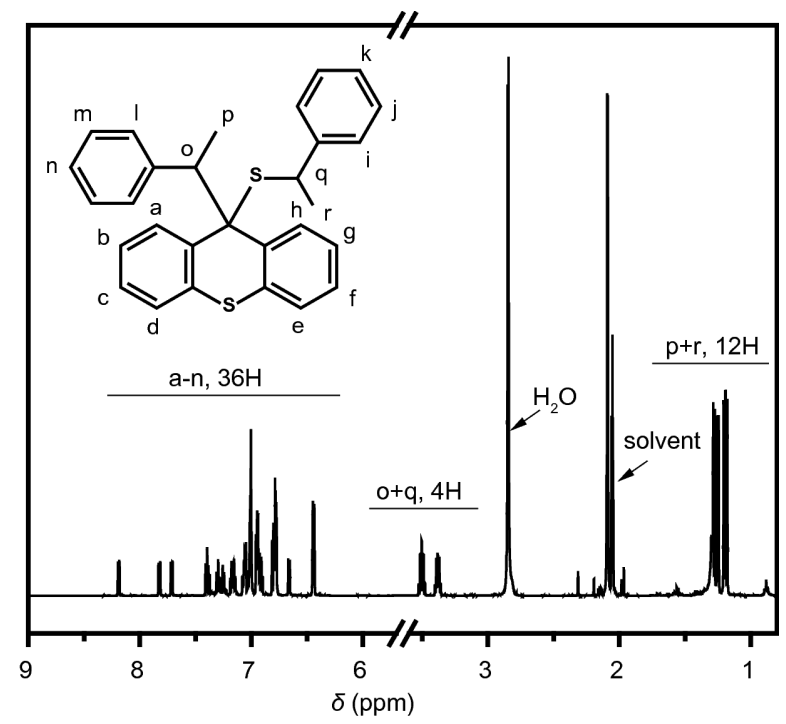

图 2 DPETTX的 ${ }^{1} \mathrm{H}$ NMR谱图 $(600 \mathrm{MHz}, \mathrm{CD} 3 \mathrm{COCD}$, TMS内标)

Figure $2{ }^{1} \mathrm{H}$ NMR spectra of DPETTX $\left(600 \mathrm{MHz}, \mathrm{CD}_{3} \mathrm{COCD}_{3}\right.$, TMS) 
由基聚合, 但引发效率较低, 且不能对分子量起到调节 作用.

在环状频哪醇自由基调节的CLRP (CMP) ${ }^{[11 \sim 13]}$ 和 氧(硫)杂曾-9-硫酮调节的CLRP方法中，在 $50^{\circ} \mathrm{C} \sim 80^{\circ} \mathrm{C}$ 之间增长链自由基和带有氧(硫)杂葱片段的叔碳自由 基之间可建立起有效的偶合/断裂平衡，使聚合物链可 控增长(图4)。当DPETTX单独引发St聚合时，小分子 DPETTX具有更高的稳定性，其在聚合温度下与聚合 物链相对应的1-苯乙基很难按照图5中式(1)所示的机 制发生有效断裂，使得体系中累积的1-苯乙基自由基



和PETTX自由基的浓度均非常低，在 $50^{\circ} \mathrm{C} \sim 80^{\circ} \mathrm{C}$ 温度 范围不能引发 $\mathrm{St}$ 聚合. 在高温下 $\left(\right.$ 如 $\left.100^{\circ} \mathrm{C}\right)$ 引发聚合的 可能机理是如图5中式(2)所示的DPETTX分子中9位C 原子和 $\mathrm{S}$ 原子之间单键的断裂 ${ }^{[19]}$, 产生的碳自由基和硫 自由基均能引发 $\mathrm{St}$ 的自由基聚合, 但会失去可控性, 导 致PS的 $M_{\mathrm{n}}$ 远高于设计分子量, 且随转化率增加不能可 控增长.

\subsection{DPETTX和ABVN共同引发St自由基聚合}

基于DPETTX对St聚合的引发效果和机理分析,

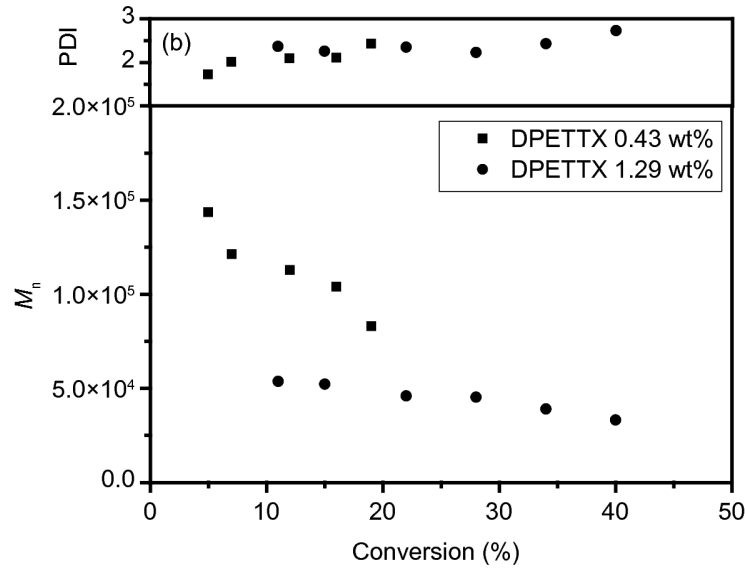

图 3 不同DPETTX浓度下St聚合的转化率-时间(a)和 $M_{\mathrm{n}}$-转化率及PDI-转化率曲线(b). 溶剂: 甲苯; 单体浓度为 $30 \mathrm{wt} \%$; 温度 为 $100^{\circ} \mathrm{C}$

Figure 3 Plot of conversion vs. time (a) and plots of $M_{\mathrm{n}} \&$ PDI vs. conversion (b) for the polymerization of St with different concentrations of DPETTX. Solvent is toluene; concentration of St is $30 \mathrm{wt} \%$; temperature is $100{ }^{\circ} \mathrm{C}$.

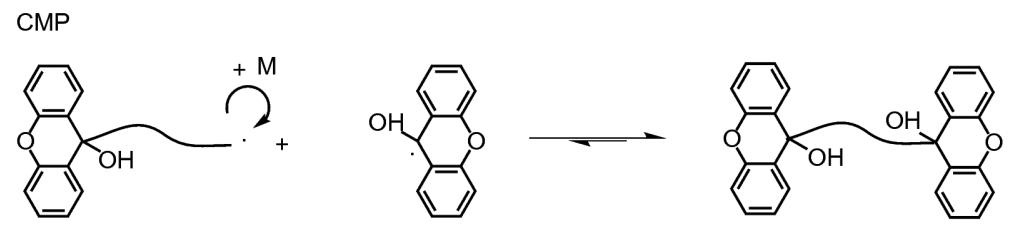

Xanthene-9-thione (thioxanthene-9-thione) mediated radical polymerization

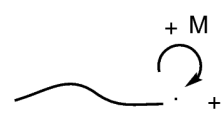<smiles>S=c1c2ccccc2oc2ccccc12</smiles><smiles>CCC</smiles><smiles>CCCSC1c2ccccc2Oc2ccccc21</smiles>

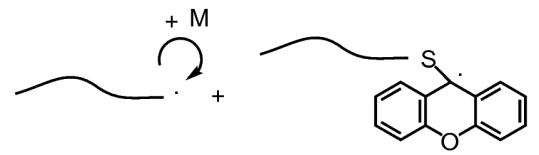
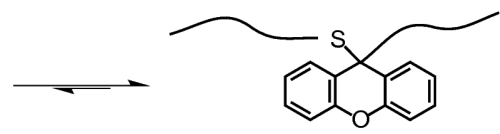

(3)

图 4 CMP和氧(硫)杂蒽-9-硫酮调节的CLRP中的可逆钝化-活化平衡

Figure 4 Reversible deactivation-activation equilibrium of CMP and xanthene-9-thione (thioxanthene-9-thione) mediated CLRP. 
我们考虑向聚合体系中引入一低温热分解引发剂 $\operatorname{ABVN}\left(55^{\circ} \mathrm{C}\right.$ 和 $65^{\circ} \mathrm{C}$ 下半衰期分别为 5 和 $\left.1 \mathrm{~h}\right)$, 以期在低 温下既能避免DPETTX分解副反应, 又能向聚合体系 中提供额外的自由基, 促使DPETTX分解并可逆钝化 链自由基, 使聚合物分子链按CMP机制增长.

我们固定DPETTX的浓度为 $1.08 \mathrm{wt} \%$, 对在 $55^{\circ} \mathrm{C}$ 下ABVN与DPETTX的摩尔比为 $0.2 / 1 、 0.1 / 1$ 和 $0.05 / 1$ 时 $\mathrm{St}$ 的聚合行为进行了研究, 结果如图6所示. 由图6(a) 可以看出, 添加少量ABVN后, 在远低于DPETTX单独 引发 $\mathrm{St}$ 聚合的温度 $\left(55^{\circ} \mathrm{C}\right)$ 下, $\mathrm{St}$ 可以有效聚合. 在 $\mathrm{ABVN} / \mathrm{DPETTX}=0.2 / 1$ 时, $12 \mathrm{~h}$ 的St转化率达到 $27 \%$,
而 $48 \mathrm{~h}$ 的St转化率则达到 $39 \%$. 随ABVN添加量下降, St转化率有所下降, 在ABVN/DPETTX为 $0.1 / 1$ 和 $0.05 / 1$ 时, $12 \mathrm{~h}$ 的St转化率分别为 $24 \%$ 和 $16 \%$, 而 $48 \mathrm{~h}$ 的St转化 率则分别为 $33 \%$ 和 $27 \%$.

图6(b)则给出了添加不同比例的ABVN时, PS的 $M_{\mathrm{n}}$ 和 P D I 随转化率的变化情况. 当 $\mathrm{A} \mathrm{B} \mathrm{V} \mathrm{N/}$ DPETTX $=0.2 / 1$ 时, $M_{\mathrm{n}}$ 从 1.5 万增长到 2.3 万, $\mathrm{PDI}$ 从 1.66 增加到2.18. 当 $\mathrm{ABVN} / \mathrm{DPETTX}=0.1 / 1$ 时, $M_{\mathrm{n}}$ 从 2.0 万增 长到 3.5 万, $\mathrm{PDI}$ 从 1.63 增加到 2.19 . 而当进一步降低 $\mathrm{ABVN}$ 用量，使 $\mathrm{ABVN} / \mathrm{DPETTX}=0.05 / 1$ 时，其 $M_{\mathrm{n}}$ 从 2.7 万增长到 4.6 万, PDI虽然从1.75逐渐增加到 2.32 , 但明

Expected mechanism of thermal decomposition
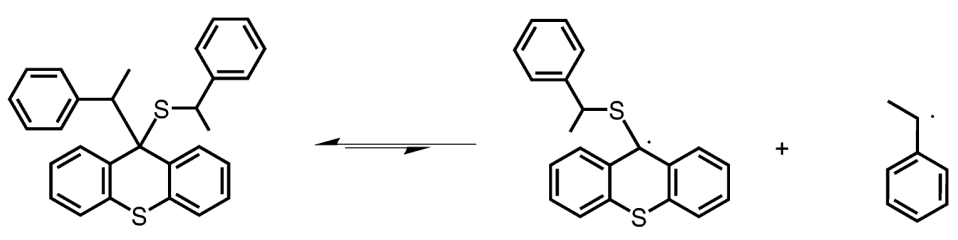

Possible mechanism of thermal decomposition at high temperature
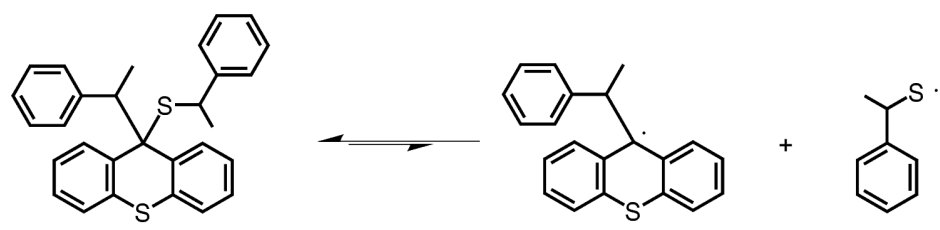

图 5 DPETTX的热分解机制

Figure 5 Thermal decomposition mechanism of DPETTX.
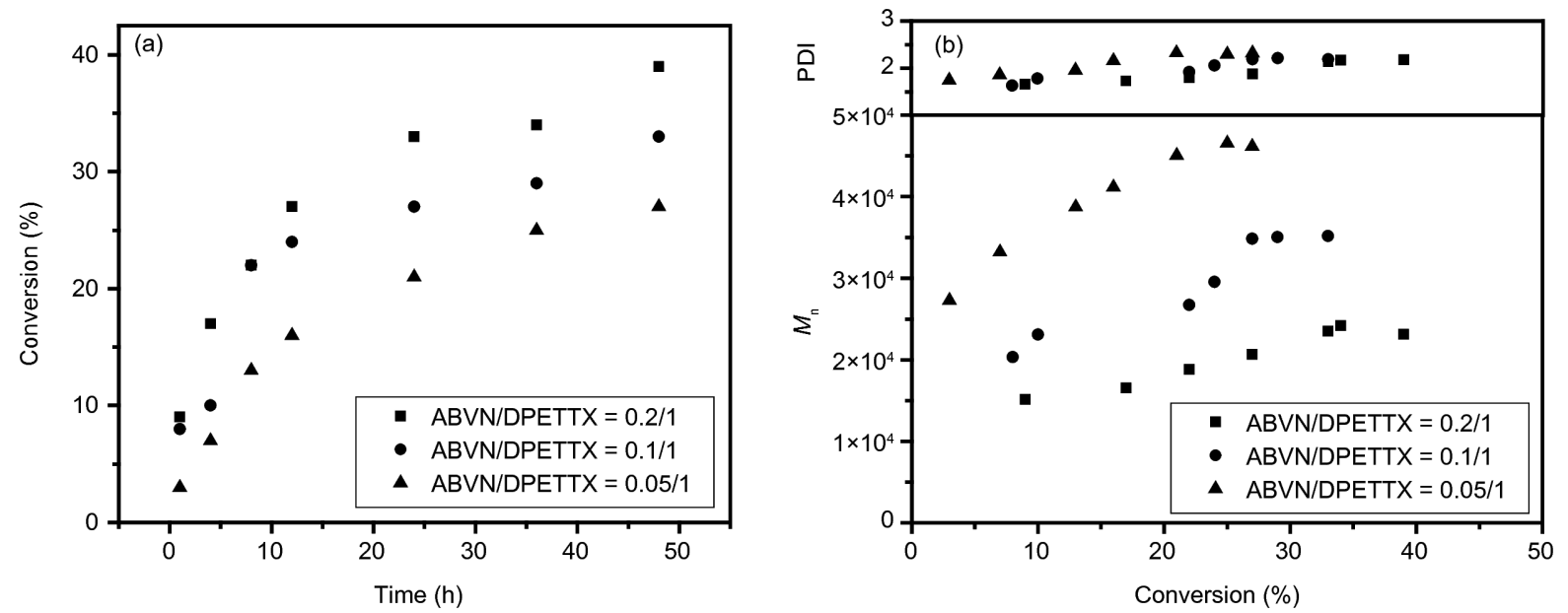

图 6 不同 $\mathrm{ABVN} / \mathrm{DPETTX}$ 摩尔比时, $\mathrm{St}$ 聚合的转化率-时间(a)和 $M_{\mathrm{n}}$-转化率及PDI-转化率曲线(b). 溶剂为甲苯; 单体浓度为 $30 \mathrm{wt} \%$; 温度为 $55^{\circ} \mathrm{C}$

Figure 6 Plot of conversion vs.time (a) and plots of $M_{\mathrm{n}} \&$ PDI vs. conversion (b) for the polymerization of St with different molar ratios of ABVN to DPETTX. Solvent is toluene; concentration of $\mathrm{St}$ is $30 \mathrm{wt} \%$; temperature is $55^{\circ} \mathrm{C}$. 


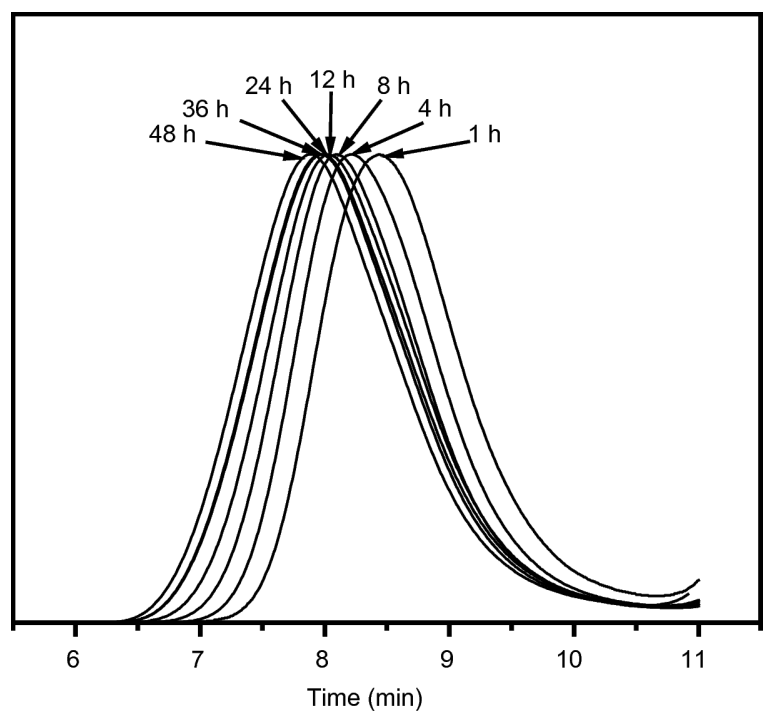

图 $755^{\circ} \mathrm{C}$ 下, $\mathrm{ABVN} / \mathrm{DPETTX}=0.05 / 1$ 时, $\mathrm{St}$ 聚合的 $\mathrm{GPC}$ 流 出曲线. 溶剂为甲苯; 单体浓度为 $30 \mathrm{wt} \%$

Figure 7 GPC traces of the polymerization of St with the molar ratio of ABVN to DPETTX being $0.05 / 1$ at $55^{\circ} \mathrm{C}$. Solvent is toluene; concentration of St is $30 \mathrm{wt} \%$.

显低于高温下DPETTX单独引发St聚合时的数值. 图7 给出了 $\mathrm{ABVN} / \mathrm{DPETTX}=0.05 / 1$ 时的 GPC流出曲线, 可 见均为单峰，且随着聚合反应的进行，其向高分子量 (更低流出时间)的方向移动，表明PS链在聚合过程中 不断增长.

这些结果表明，添加ABVN后聚合体系呈现出一 定的可控性. ABVN越多, St聚合越快, PS初始分子量
越低, 分子量增幅越小; 反之, St聚合越慢, St初始分子 量越高, 分子量增幅越大; 其分子量分布虽然较宽, 但 明显低于高温下DPETTX单独引发 $\mathrm{St}$ 聚合的数值. 这 些结果和规律可以简单地理解为在此低温下, 聚合主 要来自于 $\mathrm{ABVN}$ 的分解, 因此ABVN越多, 聚合越快, 初始PS分子量越低; 而PS链的增长取决于由PS链自由 基与DPETTX分解后形成的交叉休眠种，在DPETTX 浓度固定时，ABVN越少，该类“活性”休眠端基越少， 导致分子量增长幅度越大; 由于两种聚合方式共存, 导致PS整体分子量分布明显宽于其他活性聚合体系.

基于这种认识，我们在 $\mathrm{ABVN} / \mathrm{DPETTX}=0.05 / 1$ 的 条件下，观测了不同聚合温度时 St的聚合行为，如图8 所示. 从图8(a)可以看出, 随着聚合温度升高, $\mathrm{St}$ 聚合速 率有小幅上升. 在 $60^{\circ} \mathrm{C}$ 下 $12 \mathrm{~h}$ 时的 $\mathrm{St}$ 转化率为 $17 \%$, $48 \mathrm{~h}$ 时的 $\mathrm{St}$ 转化率为 $29 \%$, 略高于 $55^{\circ} \mathrm{C}$ 下的对应值(图6 (a)). 当温度升高到 $65^{\circ} \mathrm{C}$ 时, $12 \mathrm{~h}$ 时的 $\mathrm{St}$ 转化率为 $18 \%$, 而 $48 \mathrm{~h}$ 的St转化率则为 $31 \%$. 进一步提高聚合温度至 $70^{\circ} \mathrm{C} ， 12 \mathrm{~h}$ 的St转化率小幅升高至 $20 \%$ ，而 $48 \mathrm{~h}$ 的 St转 化率则升高至 $33 \%$.

图8(b)给出了不同聚合温度下PS的 $M_{\mathrm{n}}$ 和PDI随转 化率的变化情况. 在 $60^{\circ} \mathrm{C}$ 下PS的 $M_{\mathrm{n}}$ 从 2.6 万升高到 4.6 万, $\mathrm{PDI}$ 从1.74升高到 2.19 . 当温度升高到 $65^{\circ} \mathrm{C}$ 时, $\mathrm{PS}$ 的 $M_{\mathrm{n}}$ 从 2.5 万升高到 4.1 万, PDI从 1.74 升高到 2.29 . 进一步 提高聚合温度至 $70^{\circ} \mathrm{C}, \mathrm{PS}$ 的 $M_{\mathrm{n}}$ 从 2.3 万升高到 3.8 万, PDI从1.85升高到2.32. 这些表明随着转化率的增加, PS链不断增长.
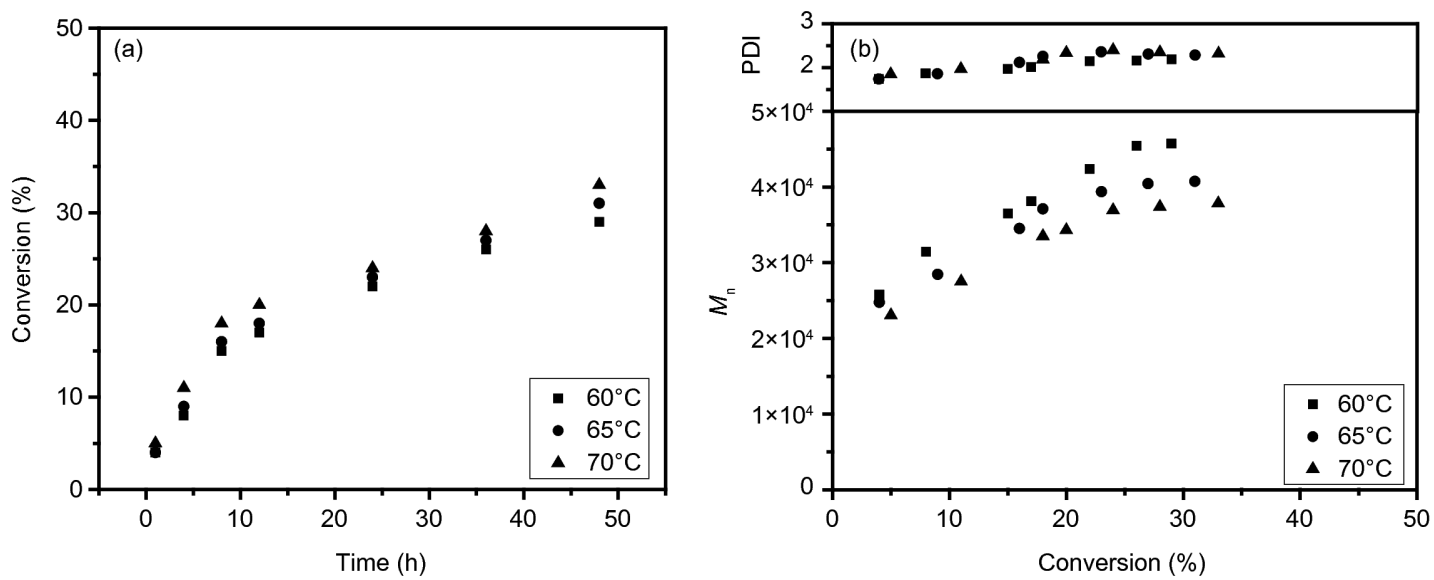

图 8 不同温度下, $\mathrm{ABVN} / \mathrm{DPETTX}=0.05 / 1$ 时, $\mathrm{St}$ 聚合的转化率-时间 $(\mathrm{a})$ 和 $M_{\mathrm{n}}$-转化率及PDI-转化率曲线(b). 溶剂为甲苯; 单体 浓度为 $30 \mathrm{wt} \%$

Figure 8 Plot of conversion vs.time (a) and plots of $M_{\mathrm{n}}$ \& PDI vs. conversion (b) for the polymerization of St with the molar ratio of ABVN to DPETTX being $0.05 / 1$ at different temperatures. Solvent is toluene; concentration of St is $30 \mathrm{wt} \%$. 


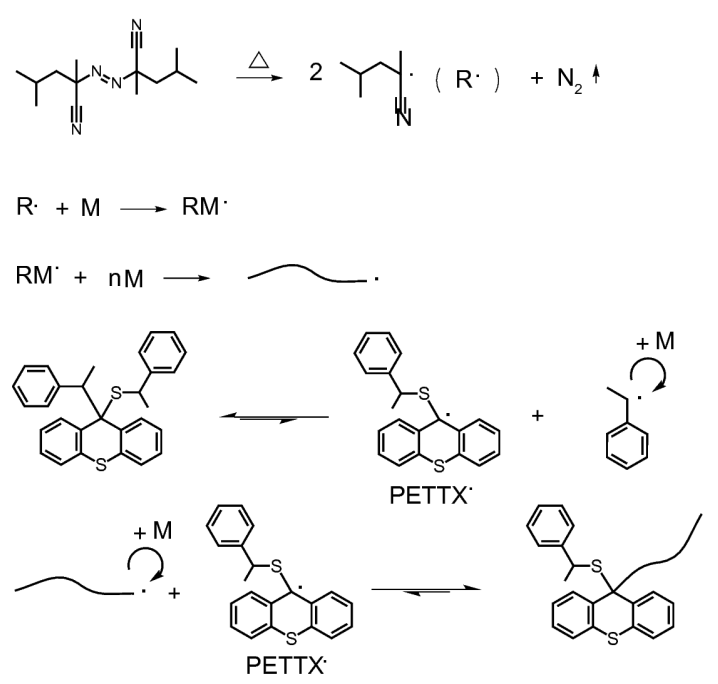

图 9 DPETTX和ABVN共同引发St聚合可能的机理示意图 Figure 9 Possible mechanism of the polymerization of St co-initiated by DPETTX and ABVN.

以上结果表明，在 $A B V N / D P E T T X=0.05 / 1$ 的条件 下，不同聚合温度下 $\mathrm{St}$ 的聚合均保持着一定的可控性. 随着聚合温度的升高, 聚合速率小幅上升, PS的初始 分子量降低, 且随转化率升高, $M_{\mathrm{n}}$ 的增长幅度有所下 降. 可以理解为随温度升高, ABVN的分解速率和链增 长速率加快, 聚合速率加快, PS的初始分子量降低. ABVN分解越快, PS链自由基与DPETTX分解产物形 成的“活性”休眠端基越多，导致温度升高时 $M_{\mathrm{n}}$ 的增长 幅度减小.

基于以上实验结果和分析，我们提出了DPETTX 和ABVN共同引发 $\mathrm{St}$ 聚合可能的机理, 如图9所示. 当
添加少量ABVN时, ABVN均裂产生两个初级自由基 (图9式(1)), 经链引发和链增长得到链自由基(式(2, 3)). 这些链自由基可以和DPETTX断裂(式(4))产生的 PETTX自由基发生可逆的偶合-断裂反应(式(5)), 并促 使式(4)所示的平衡反应不断向右进行. 通过这种方式, 使得聚合体系中积累了足够的链自由基和PETTX自 由基. 这一方面保证了 $\mathrm{St}$ 在 $55^{\circ} \mathrm{C}$ 下也能实现有效聚合, 另一方面PETTX自由基和链自由基建立类似CMP机 理的可逆偶合/断裂平衡, 使得PS的链增长得到有效控 制，所得PS的分子量随转化率增加而不断增长. 由于 该体系中用于可逆钝化链自由基的PETTX自由基初 始浓度很低, 因此聚合初始阶段PS的 $M_{\mathrm{n}}$ 较高, 这与 CMP体系类似. 而所得PS的PDI较高, 则是因为链自由 基不可逆的终止反应以及DPETTX可能的副反应.

\section{4 结论}

采用 $\mathrm{Cu} / \mathrm{PMDETA}$ 催化硫杂葱-9-硫酮和1-溴苯乙 烷的偶联反应, 成功制备了DPETTX. DPETTX在高温 $\left(100^{\circ} \mathrm{C}\right)$ 下可单独引发 $\mathrm{St}$ 聚合, 但引发效率较低. 当其与 少量 $\mathrm{ABVN}$ 共用时，可使 $\mathrm{St}$ 在低温下 $\left(55^{\circ} \mathrm{C}\right)$ 聚合， $\mathrm{PS}$ 的 分子量随转化率升高而不断增大; 随着 $\mathrm{ABVN}$ 添加量 的增加, 聚合反应速率不断增大, 且聚合过程中产物 $M_{\mathrm{n}}$ 的增加幅度下降; 提高反应温度, PS的分子量增加 幅度下降. 该基于DPETTX和少量ABVN的共引发体 系，打开了一条开发可控/活性自由基聚合体系的新 路径.

\section{补充材料}

本文的补充材料见网络版http://chemcn.scichina.com. 补充材料为作者提供的原始数据, 作者对其学术质量和内容负责.

\section{参考文献}

1 Matyjaszewski K, Tsarevsky NV. J Am Chem Soc, 2014, 136: 6513-6533

2 Georges MK, Veregin RPN, Kazmaier PM, Hamer GK. Macromolecules, 1993, 26: 2987-2988

3 Hawker CJ. J Am Chem Soc, 1994, 116: 11185-11186

4 Wang JS, Matyjaszewski K. J Am Chem Soc, 1995, 117: 5614-5615

5 Kato M, Kamigaito M, Sawamoto M, Higashimura T. Macromolecules, 1995, 28: 1721-1723

6 Chiefari J, Chong YKB, Ercole F, Krstina J, Jeffery J, Le TPT, Mayadunne RTA, Meijs GF, Moad CL, Moad G, Rizzardo E, Thang SH. Macromolecules, 1998, 31: 5559-5562 
7 Percec V, Guliashvili T, Ladislaw JS, Wistrand A, Stjerndahl A, Sienkowska MJ, Monteiro MJ, Sahoo S. J Am Chem Soc, 2006, 128: 1415614165

8 Yamago S, Iida K, Yoshida J. J Am Chem Soc, 2002, 124: 2874-2875

9 Yamago S, Ray B, Iida K, Yoshida JI, Tada T, Yoshizawa K, Kwak Y, Goto A, Fukuda T. J Am Chem Soc, 2004, 126: 13908-13909

10 Yamago S, Kayahara E, Kotani M, Ray B, Kwak Y, Goto A, Fukuda T. Angew Chem Int Ed, 2007, 46: 1304-1306

11 Zheng X, Yue M, Yang P, Li Q, Yang W. Polym Chem, 2012, 3: 1982-1986

12 Huang X, Wang L, Yang W. Polym Chem, 2015, 6: 6664-6670

13 Yao CR, Wang L, Yang WT. RSC Adv, 2016, 6: 69743-69747

14 Rizzardo E, Solomon DH. Polym Bull, 1979, 1: 529-534

15 Toy AA, Chaffey-Millar H, Davis TP, Stenzel MH, Izgorodina EI, Coote ML, Barner-Kowollik C. Chem Commun, 2006, 58: 835-837

16 Junkers T, Stenzel MH, Davis TP, Barner-Kowollik C. Macromol Rapid Commun, 2007, 28: 746-753

17 Günzler F, Junkers T, Barner-Kowollik C. J Polym Sci A, 2009, 47: 1864-1876

18 Junkers T, Delaittre G, Chapman R, Günzler F, Chernikova E, Barner-Kowollik C. Macromol Rapid Commun, 2012, 33: 984-990

19 Li XE. Fractural carbon-carbon bond radical initiators: design, synthesis and application. Dissertation for the Doctoral Degree. Beijing: Beijing University of Chemical Technology, 2014. 23-120 (in Chinese) [李雪娥. 几种C-C键断裂自由基引发剂的设计合成和应用的初步研究. 博士 学位论文. 北京: 北京化工大学, 2014. 23-120]

\title{
Radical polymerization of styrene mediated by 9-(1-phenylethyl)-9- ((1-phenylethyl)thio)-9H-thioxanthene
}

\author{
Shichao Wang ${ }^{1,2}$, Li Wang ${ }^{1,2}$, Haoyu Yu ${ }^{1,2}$, Jianwei Shao ${ }^{1,2}$, Wantai Yang ${ }^{1, *^{*}}$ \\ ${ }^{1}$ State Key Laboratory of Chemical Resource Engineering, Beijing University of Chemical Technology, Beijing 100029, China \\ ${ }^{2}$ College of Materials Science and Engineering, Beijing University of Chemical Technology, Beijing 100029, China \\ *Corresponding author (email: yangwt@mail.buct.edu.cn)
}

\begin{abstract}
Rapid progress in controlled/living radical polymerization (CLRP) has been achieved since the end of the last century. However, the demerits and limitations of existed methods impede their industrial applications on a large scale. Therefore, it is prominent to develop new CLRP methods that are better in controlled/living features, more simple, feasible and environmental-friendly. In this article, a new thioether, 9-(1-phenylethyl)-9-((1-phenylethyl)thio)-9Hthioxanthene (DPETTX) was synthesized. Then, the polymerization behavior of St initiated by DPETTX and both DPETTX and a small amount of 2,2'-azobisisoheptonitrile (ABVN) was studied. Results show that DPETTX can initiated the polymerization of $\mathrm{St}$ at a high temperature $\left(100^{\circ} \mathrm{C}\right)$ with low initiation efficiency, while St polymerization at a low temperature $\left(55^{\circ} \mathrm{C}\right)$ was possible in the presence of a small amount of ABVN and number average molecular weight $\left(M_{\mathrm{n}}\right)$ increased continuously with increasing conversion of St. $M_{\mathrm{n}}$ increased from $27 \mathrm{k}$ to $46 \mathrm{k}$ when a molar ratio of ABVN/DPETTX $=0.05 / 1$ was adopted. This co-initiating system based on DPETTX and a small amount of ABVN paves a new way for developing new CLRP methods.
\end{abstract}

Keywords: thioether, radical, polymerization, molecular weight

doi: $10.1360 / \mathrm{N} 032017-00204$ 